\title{
Error propagation dynamics of PIV-based pressure field calculation (3): What is the minimum resolvable pressure in a reconstructed field?
}

\author{
Zhao Pan ${ }^{1 *}$, Jared P. Whitehead ${ }^{3}$, Geordie Richards ${ }^{2}$, Barton L. Smith ${ }^{2 \dagger}$ \\ ${ }^{1}$ University of Waterloo, Department of Mechanical and Mechatronics Engineering, ON N2L 3G1, Canada \\ ${ }^{2}$ Utah State University, Mechanical and Aerospace Engineering, Logan, UT 84322, USA \\ ${ }^{3}$ Brigham Young University, Mathematics Department, Provo, UT 84602, USA \\ *zhao.pan@uwaterloo.ca † barton.smith@usu.edu
}

\begin{abstract}
An analytical framework for the propagation of velocity errors into PIV-based pressure calculation is established. Based on this framework, the optimal spatial resolution and the corresponding minimum field-wide error level in the calculated pressure field are estimated. This minimum error is viewed as the smallest resolvable pressure. We find that the optimal spatial resolution is a function of the flow features, geometry of the flow domain, and the type of the boundary conditions, in addition to the error in the PIV experiments, making a general statement about pressure sensitivity is difficult. The minimum resolvable pressure is affected by competing effects from the experimental error due to PIV and the truncation error from the numerical solver. This means that PIV experiments motivated by pressure measurements must be carefully designed so that the optimal resolution (or close to the optimal resolution) is used. Flows $\left(\mathrm{Re}=1.27 \times 10^{4}\right.$ and $5 \times 10^{4}$ ) with exact solutions are used as examples to validate the theoretical predictions of the optimal spatial resolutions and pressure sensitivity. The numerical experimental results agree well with the analytical predictions.
\end{abstract}

\section{Introduction}

Experimental pressure measurements are useful for determining loads on structures and for examination of acoustic effects. However, it has not been possible to measure the pressure field away from surfaces until recently. In the last decades, it has been shown that the pressure field may be determined from velocity data measured using Particle Image Velocimetry (PIV). PIV-based pressure calculation techniques have received significant recent interest because the output provides field measurements with high frequency response (Van Oudheusden, 2013). The accuracy of pressure fields derived from PIV data has improved with the PIV technique itself, as hardware improvements provide increased spatial and temporal resolution and new techniques have provided a fully three-dimensional velocity field (Scarano, 2012). With these recent advances, a natural inquiry is "How accurate is the pressure field obtained from PIV?" or "How do errors in the velocity field affect the errors of the computed pressure field?" or "What is the minimum measurable pressure?" This work will attempt to address these questions. But first we briefly survey the state-of-the-art in PIV-based pressure field calculations.

Velocimetry-based pressure reconstruction is a straight-forward idea that can be traced back to Schwabe (1935). Technical limitations of the imaging technique in Schwabe (1935) (i.e., low spatial and temporal resolution, etc.) and consequently large error in the velocity field measurement, led to a calculated pressure that was not reliable enough to ensure any quantitative confidence at the time. After decades of development, PIV has become a reliable non-invasive velocity field measurement technique, which not only provides the vector velocity field measurements but also describes the uncertainty of these measurements (Sciacchitano et al., 2013; Charonko and Vlachos, 2013; Wieneke, 2015).

Built on these advancements in PIV techniques, calculation of the PIV-based pressure field has become common. PIV-based pressure field reconstruction methods are divided into two categories: i) integrating the 
pressure gradient from the Navier-Stokes equations, and ii) integrating the Poisson equation derived from the Navier-Stokes equations to obtain the scalar pressure field. Recent algorithmic considerations investigate alternative 'numerical methods' such as a least square solver (Jeon et al., 2015), spectral decomposition (Wang et al., 2017), and data assimilation (e.g., He et al. (2020)) to achieve a robust or fast pressure solution. Pressure-Poisson-equation-based methods often involve well-defined explicit boundary conditions, which have a straightforward physical and mathematical interpretation. Examples may be found in, for example, de Kat and Van Oudheusden (2012); Pröbsting et al. (2013). For either the pressure gradient integration or the Poisson equation approach, the state-of-the-art implementation uses time-resolved 2D and/or 3D PIV data and numerically optimized solvers.

Taking advantage of the advancing velocimetry techniques and velocimetry-based pressure reconstruction algorithms, PIV-based pressure reconstruction techniques have been increasingly applied to various fields of study. Examples in classic topics include pressure field and loads on airfoils (Jeon et al., 2016; Lignarolo et al., 2014), water slamming pressure of boat hull (Porfiri and Shams, 2017), as well as pressure distribution in turbulent boundary layers (Ghaemi et al., 2012; Zhang et al., 2017). The extended applications cover aero-acoustics with acoustic analogies (Léon et al., 2017), and compressible flows Van Oudheusden et al. (2007); Van Gent et al. (2018).

Fundamental research on PIV-based pressure calculations has shed insight into design of experiments and algorithms. For example, Charonko et al. (2010) benchmarked several different pressure field reconstruction algorithms and found that the performance of the PIV-based pressure calculations are affected by almost every factor involved in the experiments (e.g., type of flow, spatial and temporal resolutions, filtering of the PIV data, the type of numerical solver, and error level in the PIV data). There is no universal optimal experimental setup for all applications, although, as shown below for a specific problem, optimal spatial and temporal resolutions do exist that minimize the error in the calculated pressure field. de Kat and Van Oudheusden (2012) pointed out that a numerical Poisson solver acts as a low-pass filter, which tends to eliminate the high frequency data (both signal and noise) from the PIV experiments. In subsequent work, Matthew Faiella and Pan (2021) showed that this low-pass filter is not only due to the specific numerical scheme of the Poisson filter, but is rooted in the properties of the Poisson operator. Thus, low frequency error due to PIV measurements should be avoided to minimize the error that propagates to the calculated pressure. A more general study of error propagation of the PIV-based pressure field calculation showed that the fundamental features of the domain such as geometry (dimension, shape, and size) of the domain and the configuration of boundary conditions impact the error propagation as well (Pan et al., 2016).

Despite numerous recent studies on PIV-based pressure calculations as a quantitative measurement technique, the uncertainty of the technique and how it depends on the accuracy of the velocity measurement has not been sufficiently addressed. Only a few works have covered this topic. Azijli et al. (2016) proposed a posteriori uncertainty quantification method of PIV-based pressure calculations under a Bayesian framework. Pan et al. (2016) introduced an upper bound on the error in the calculated pressure field which is a function of some fundamental factors of the flow field, such as geometry of the domain and the type of boundary conditions. Even though the upper bound is not always apparent and could overestimate the error, it can be considered an a priori estimate of the worst possible error level in the reconstructed pressure field and thus aid the experimental design and optimization. More recently, (McClure and Yarusevych, 2017) proposed an estimation of the optimal spatial and temporal resolution that minimize the error in the pressure field, but did not provide the minimum error in the reconstructed pressure, which can be interpreted as the sensitivity of the pressure reconstruction.

In the current study, we will begin to answer one of the fundamental questions posed above: "What is the minimum resolution or the sensitivity of the PIV-based pressure calculation for a given experimental setup, and what is the optimal spatial resolution for a PIV experiment with pressure reconstruction being the end goal?" Flows with exact solutions will be used for validation. Based on analytical predictions, practical solutions will be given for real engineering applications.

\section{Problem setup and definitions}

PIV-based pressure calculation is rooted in the Navier-Stokes equations. Arranging the nondimensionalized Navier-Stokes equations we have $\nabla p=-\left(\frac{\partial u}{\partial t}+(\boldsymbol{u} \cdot \nabla) \boldsymbol{u}-\frac{1}{R e} \nabla^{2} \boldsymbol{u}\right)$, where $\boldsymbol{u}$ is the velocity field, which is obtained from experiments, and $p$ is the pressure field, which is to be determined. $R e$ is the Reynolds number. As noted above, current PIV-based pressure field calculation methods fall into two categories: i) direct integration of the pressure gradient $(\nabla p)$ (e.g., Liu and Katz (2006)) from the Navier-Stokes equations, ii) applying the divergence operator to $\nabla p$ and solving the corresponding Poisson equation with respect to 
the pressure field $p$ :

$$
\nabla^{2} p=f(\boldsymbol{u})=-\nabla \cdot\left(\frac{\partial \boldsymbol{u}}{\partial t}+(\boldsymbol{u} \cdot \nabla) \boldsymbol{u}-\frac{1}{R e} \nabla^{2} \boldsymbol{u}\right),
$$

where the right hand side, $f(\boldsymbol{u})$, is called the "data" (see Pan et al. (2016)). We will adopt this terminology hereafter in the current work. In this study, we focus on the latter method. To prevent any confusion, we will address the experimental data from PIV as "experimental results" or "PIV results". Eq. (1) must be solved with proper boundary conditions (BCs). A complete description of the problem in a domain $\Omega$ can be described as $\nabla^{2} p=f(\boldsymbol{u})$ in $\Omega$, with Neumann BCs $\nabla p \cdot \mathbf{n}=g(\boldsymbol{u})$ on $\partial \Omega$ (enforced pressure gradient on the boundary), and/or Dirichlet BCs $p=h(\boldsymbol{u})$ on $\partial \Omega$ (enforced pressure on the boundary), where $f(\boldsymbol{u}), g(\boldsymbol{u})$ and $h(\boldsymbol{u})$ are corresponding functions of the velocity field.

Clearly, the error from experimental measurements will propagate to the calculated pressure field. However, the typical propagation analysis using Taylor series method or Monte Carlo methods (Coleman and Steele, 2009) are difficult. In the current study, we directly analyze the error propagation of the PIV-based pressure calculation using the underlying governing equations (1) and corresponding boundary conditions. Denoting the error in the measured velocity field as $\boldsymbol{\varepsilon}_{u}$ and the true value of the velocity field as $\boldsymbol{u}$, the error contaminated velocity measurement $\tilde{\boldsymbol{u}}$ can be modeled as $\tilde{\boldsymbol{u}}=\boldsymbol{u}+\boldsymbol{\varepsilon}_{u}$. Similarly, the pressure field with errors $\tilde{p}$ can be modeled as $\tilde{p}=p+\varepsilon_{p}$, where $\varepsilon_{p}$ is the error in the calculated pressure field and $p$ is the unknown true value. With measurement error considered, the pressure field reconstruction problem are implemented, in practice, as $\nabla^{2} \tilde{p}=f(\tilde{\boldsymbol{u}})$ in $\Omega$, with Neumann BCs, $\nabla \tilde{p} \cdot \mathbf{n}=g(\tilde{\boldsymbol{u}})$ on $\partial \Omega$, and/or Dirichlet BCs $\tilde{p}=h(\tilde{\boldsymbol{u}})$ on $\partial \Omega$. We will further quantify the relationship between $\boldsymbol{\varepsilon}_{u}$ and $\varepsilon_{p}$. To adequately perform this comparison, we use the space-averaged $L^{2}$-norm of a field on the domain $\Omega$ defined as $\left.|| \varepsilon\right|_{L^{2}(\Omega)}=\sqrt{\int \varepsilon^{2} d \Omega} /|\Omega|$, where $|\Omega|$ denotes the area or volume of the domain depending on the dimensions of the domain.

\section{Error estimation of reconstructed pressure field}

In practice, a PIV experiment is associated with spatial and temporal resolutions. Solving pressure field from PIV results is often numerically achieved and involves three important factors: spatial resolution, temporal resolution, and the numerical scheme of the pressure solver. In the current work, we focus on an analytical investigation on the impact of spatial resolution of PIV experiments on the error propagation of PIV-based pressure field reconstruction.

\subsection{Theory: error propagation, minimum error, and optimal spatial resolution}

We consider a two dimensional (2D) flow on a structured mesh with grid spacing $h \times h$. We assume that the measured velocity field from the PIV experiments has point-wise zero-mean Gaussian noise with variance $\sigma_{u}$ and $\sigma_{v}$ in the two cardinal directions. The expected error level in the calculated pressure field can be estimated as

$$
\begin{aligned}
\left\|\varepsilon_{p}\right\|_{\left(L^{2}(\Omega)\right)} & \lesssim\left\|\varepsilon_{p, T}\right\|_{L^{2}(\Omega)}+\left\|\varepsilon_{p, E}\right\|_{L^{2}(\Omega)} \\
& \approx \underbrace{C_{1}\left(\left\|\frac{\partial^{2} p}{\partial x^{2}}\right\|_{L^{2}(\Omega)}+2\left\|\nabla^{-2} \frac{\partial^{4} p}{\partial^{2} x \partial^{2} y}\right\|_{L^{2}(\Omega)}+\left\|\frac{\partial^{2} p}{\partial x^{2}}\right\|_{L^{2}(\Omega)}\right)}_{\text {Truncation error contribution }} h^{m}+\overbrace{C_{0} C_{2} \frac{\sigma_{u}^{2}+\sigma_{v}^{2}}{2} h^{n}}^{\text {PIV error contribution }},
\end{aligned}
$$

where $\left\|\varepsilon_{p, T}\right\|_{L^{2}(\Omega)}$ is the truncation error of the numerical scheme arising from the Poisson solver, and the second term $\left(\left\|\varepsilon_{p, E}\right\|_{L^{2}(\Omega)}\right)$ includes the effect of the experimental errors in the measured velocity field (the derivation of this estimate with greater details can be found in Pan et al. (2018)). For a specific example, a flow in an $L \times L$ square domain, with pure Dirichlet boundary conditions, the pressure field is solved by a second order finite difference Poisson solver with central difference scheme. In this setting, (2) leads to a more particular form with specific parameters:

$$
\left\|\varepsilon_{p}\right\|_{\left(L^{2}(\Omega)\right)} \lesssim \frac{1}{12}\left(\left\|\frac{\partial^{2} p}{\partial x^{2}}\right\|_{L^{2}(\Omega)}+2\left\|\nabla^{-2} \frac{\partial^{4} p}{\partial^{2} x \partial^{2} y}\right\|_{L^{2}(\Omega)}+\left\|\frac{\partial^{2} p}{\partial x^{2}}\right\|_{L^{2}(\Omega)}\right) h^{2}+0.901^{2} \frac{L^{2}}{2 \pi^{2}} \frac{\sigma_{u}^{2}+\sigma_{v}^{2}}{2} h^{-2} .
$$


The physical and/or mathematical interpretations of the terms and variables in (2) or (3) can be found in Table 1.

The results above are developed for the non-dimensional setup. The dimensional equivalent estimates can be recovered by multiplying the variables by corresponding characteristic scales (e.g., $\left\|\varepsilon_{p}^{*}\right\|_{L^{2}(\Omega)}=$ $\left\|\varepsilon_{p}\right\|_{L^{2}(\Omega)} P_{0}, x^{*}=x L_{0}, u^{*}=u U_{0}$, etc., where $P_{0}, L_{0}$ and $U_{0}$ are characteristic pressure, length, and velocity respectively). For convenience, the superscript $\left([]^{*}\right)$ denoting dimensional variables will be dropped here after without special note and the non-dimensional variables will be written explicitly (e.g., $p / P_{0}$ is the non-dimensional pressure, where $p$ is the corresponding dimensional variable).

Table 1: Variables and terms in (2) and the corresponding specific values in (3) and the physi$\mathrm{cal} /$ mathematical interpretations.

\begin{tabular}{|c|c|c|c|}
\hline $\begin{array}{l}\text { Variables } \\
\text { or terms }\end{array}$ & Value & Mathematical/physical interpolation & Affected by \\
\hline$\varepsilon_{p}$ & - & Error field in the calculated pressure field & Everything \\
\hline$\left\|\varepsilon_{p}\right\|_{L^{2}(\Omega)}$ & - & Global measure of the error level of the calculated pressure field & Everything \\
\hline$C_{1}$ & $\frac{1}{12}$ & The constant of truncation error contribution & Numerical scheme \\
\hline$\frac{\partial^{2} p}{\partial x^{2}}$, etc. & - & 2nd order derivative of the pressure field & Flow field \\
\hline$C_{0}$ & $0.901^{2}$ & $\begin{array}{l}\text { Amplification ratio of point-wise Gaussian error to the "most } \\
\text { dangerous mode" of the error in the data" }\end{array}$ & $\begin{array}{l}\text { Dimension, type of BCs of } \\
\text { the domain }\end{array}$ \\
\hline$C_{2}$ & $\frac{L^{2}}{2 \pi^{2}}$ & $\begin{array}{l}\text { Optimal Poincaré constant (highest possible amplification ratio of } \\
\text { error in the reconstructed pressure field to the error in the data) }\end{array}$ & $\begin{array}{l}\text { Dimension, area, shape, } \\
\text { type of BCs }\end{array}$ \\
\hline$\sigma_{u}^{2}$, etc. & - & Variance of error of the experimental data & Quality of PIV \\
\hline$h$ & - & Spatial resolution & $\begin{array}{l}\text { PIV experiment setup and } \\
\text { post-processing }\end{array}$ \\
\hline$m$ & 2 & $\begin{array}{l}\text { Scaling constant of grid spacing for the contribution from the } \\
\text { truncation error }\end{array}$ & Numerical scheme \\
\hline$n$ & -2 & $\begin{array}{l}\text { Scaling constant of grid spacing for the contribution from the } \\
\text { experimental error }\end{array}$ & Numerical scheme \\
\hline
\end{tabular}

* Details about the derivative, calculation, and physical interpretation of $C_{0}$ can be found in Matthew Faiella and Pan (2021).

Equation (2) can be written as a function of the spatial resolution: $\left\|\varepsilon_{p}\right\|_{L^{2}(\Omega)}=$ fun $(h) \approx A h^{m}+B h^{n}$, where $A, B, m$, and $n$ are constants once the experimental setup, parameters, and pressure solver are determined. For example, for Eq. (3), $A=\frac{1}{12}\left(\left\|\frac{\partial^{2} p}{\partial x^{2}}\right\|_{L^{2}(\Omega)}+\left\|\nabla^{-2} \frac{\partial^{4} p}{\partial^{2} x \partial^{2} y}\right\|_{L^{2}(\Omega)}+\left\|\frac{\partial^{2} p}{\partial y^{2}}\right\|_{L^{2}(\Omega)}\right), B=0.901^{2} \frac{L^{2}}{2 \pi^{2}} \frac{\sigma_{u}^{2}+\sigma_{v}^{2}}{2}$, $m=2$, and $n=-2$. Clearly, $\left\|\varepsilon_{p}\right\|_{L^{2}(\Omega)}$ is not monotonic in $h$, leaving several open questions: i) what is the minimum error $\left(\left\|\varepsilon_{p}\right\|_{L^{2}(\Omega)}\right)$ ? and ii) when is the minimum approached in terms of spatial resolution $(h)$ ?

We note that $A h^{2}+B h^{-2} \geq 2 \sqrt{A B}$, where equality is reached if and only if $A h^{2}=B h^{-2}$, and we thus have the optimal spatial resolution $h^{\text {opt }} \approx \sqrt[4]{B / A}$, which leads to an estimate of the minimum error level in the calculated pressure field: $\left\|\varepsilon_{p}\right\|_{L^{2}(\Omega)}^{\min } \approx 2 \sqrt{A B}$. This minimum error level can be interpreted as the overall sensitivity of the pressure reconstruction, meaning that any pressure reconstruction results smaller than this sensitivity are not meaningful. In other words, this sensitivity of the reconstructed pressure field is a global measure of the best possible accuracy of the current PIV-based pressure reconstruction.

\subsection{Validation}

Consider a Taylor vortex in 2D. Assuming pressure at the far field vanishes $\left(p_{\infty}=0\right)$, the velocity and pressure fields are defined as $u_{\theta}(r, t)=\frac{H r}{8 \pi v t^{2}} \exp \left(-\frac{r^{2}}{4 v t}\right)$ and $p(r, t)=-\rho \frac{H r^{2}}{64 \pi^{2} v t^{3}} \exp \left(-\frac{r^{2}}{2 v t}\right)$, respectively, where $H=M / 2 \rho v$ is a constant that measures the amount of angular momentum $M$ in the vortex (Panton, 2006). The time is $t$, the distance from the center of the vortex is $r$, and $\rho$, and $v$ are density and kinematic viscosity of the fluid, respectively. We non-dimensionalize the variables as $\zeta=r / L_{0}, \xi=u / U_{0}$, and $\eta=$ $p / P_{0}$, where $L_{0}=\sqrt{2 v t}, U_{0}=H /\left(2 \pi L_{0} t\right)$, and $P_{0}=\rho U_{0}^{2}$, are the characteristic scales. Scaling $u_{\theta}$ and $p_{\theta}$ leads to $\xi_{\theta}^{*}=\frac{1}{2} \zeta \exp \left(-\frac{\zeta^{2}}{2}\right)$, and $\eta^{*}=-\frac{1}{8} \exp \left(-\zeta^{2}\right)$, respectively. 
Now we consider a 'realistic' flow in water with parameters shown in the table (see Fig. 1), and the 2D non-dimensional representation of the flow (velocity and pressure field) is also shown in the same figure. We again consider point-wise Gaussian errors added to the velocity field with zero-mean and constant standard deviation (i.e., $\left.\varepsilon_{u} \sim \mathcal{N}\left(0, \sigma_{u}^{2}\right), \varepsilon_{v} \sim \mathcal{N}\left(0, \sigma_{v}^{2}\right), \sigma_{u} / U_{0}=\sigma_{v} / U_{0} \approx 7.85 \times 10^{-3}\right)$. We refer to this numerical setup (i.e., referring the flow described in Table 2, and this specific errors) as setup 1 hereafter. We vary the spatial resolution $(h)$ of the domain and run the numerical experiments 5,000 times for each resolution. The normalized error level in the calculated pressure field $\left(\left\|\varepsilon_{p}\right\|_{\Omega\left(L^{2}\right)} / P_{0}\right)$ versus the normalized spatial resolution $\left(h / L_{0}\right)$ of the domain is shown in the box plot in Fig. 2(a). As mentioned, each box represents 5,000 independent numerical experiments. The theoretical predictions of the error level in the calculated pressure agree well with these numerical experiments. The blue dashed line (slope $=2$ ) indicates the first term in Eq. (3), which represents the contribution from the truncation error, which is affected by both the numerical schemes and the flow field. The blue dash-dot line (slope $=-2$ ), which is mainly affected by the property of the Poisson operator and the experimental error. The black line indicates the theoretical predication of the total error (see Eq. (3)) in the calculated pressure field. The intersection of the PIV error contribution (blue dash-dot line) and the truncation error contribution (blue dashed line) is marked by the blue circle indicating the optimal spatial resolution where the minimum global error in the calculated pressure field is achieved.

The minimum error in the calculated pressure field is $\left\|\varepsilon_{p}\right\|_{L^{2}(\Omega)}^{\min } / P_{0} \approx 2.35 \times 10^{-3}$ in this specific example. For a characteristic pressure $P_{0}=64.85 \mathrm{~Pa}$, the best possible sensitivity of the pressure field reconstruction is approximately $0.15 \mathrm{~Pa}$. This implies that a well designed and conducted PIV experiment with an accurate pressure solver could achieve high fidelity pressure reconstructions and rival the sensitivity of pressure sensors. Due to the 'velocity-to-pressure' computation in the PIV-pressure approach, the reconstructed pressure field is scalable with the characteristic pressure $\left(P_{0}=\rho U_{0}^{2}\right)$. This feature indicates that PIV-based pressure reconstruction techniques are particularly attractive for applications involving small pressure changes (e.g., slow air flows introduce relatively low values of $\rho$ and $U_{0}$, and thus low $P_{0}$ ), which often requires high cost instrumentally when using high-sensitivity pressure transducer arrays. For example, assuming an air flow having the same velocity field as the setup 1, the low density of the fluid media (e.g., $\left.\rho \approx 1 \mathrm{~kg} / \mathrm{m}^{3}\right)$ leads to a low characteristic pressure $\left(P_{0} \approx 0.065 \mathrm{~Pa}\right)$. The corresponding pressure measurement sensitivity in such a PIV-pressure calculation can be approximately as high as $\sim 1.5 \times 10^{-4} \mathrm{~Pa}$. Therefore, in addition to the ability to measure pressure anywhere in a flow field, pressure from PIV has the potential for superior accuracy for slow flows.

Remembering the dynamic range $(D)$ is the ratio between the maximum measurable $\left(p^{\max }\right)$ and the sensitivity $\left(\left\|\varepsilon_{p}\right\|_{L^{2}(\Omega)}^{\min }\right)$, we define the dynamic range of the PIV-based pressure calculation techniqes in the current work as $D=p^{\max } /\left\|\varepsilon_{p}\right\|_{L^{2}(\Omega)}^{\min }$ We expect that PIV-based pressure calculation techniques have following features: i) the maximum measurable pressure is determined by the velocity field and the fluid density, and is scalable to $\rho U_{0}^{2}$. In other words, $p^{\max }$ is flow dependent; ii) noting that the sensitivity of the measurement is affected by many factors (see Fig. 1 and Eq. (2)), the sensitivity is not a fixed value either; iii) thus, the PIV-based pressure reconstruction techniques has a "dynamic" dynamic range, which depends on many factors including the nature of the flow. This property is distinct to conventional pressure transducers' fixed dynamic range. The dynamic range of the PIV-based pressure reconstruction could be high if the experiment and pressure solver are carefully designed. For example, in the example presented above, the dynamic range is $D=4.3 \times 10^{5}$, which is comparable to (or even greater than) a typical pressure gauges.

Since the contribution from truncation error scales as $\left\|\varepsilon_{p, T}\right\|_{L^{2}(\Omega)} \sim O\left(h^{2}\right)$, and the contribution from measurement error in the velocity field scales as $\left\|\varepsilon_{p, E}\right\|_{L^{2}(\Omega)} \sim O\left(h^{-2}\right)$, we expect there to be a competition between the two errors in terms of the resolution $h$. This phenomena has been observed previously (Charonko et al., 2010; Pan, 2016; McClure and Yarusevych, 2017), as well as in the current study (e.g., Fig. 2(a)). In the following we provide an explicit interpretation based on a rigorous analysis (e.g., Eq. (2)). When the spatial resolution is too small (e.g., $h / L_{0} \rightarrow 0$ ), the error in the pressure is dominated by the error from the errors in the velocity field (green patched regime in Fig. 2(a)). When the spatial resolution is relatively large (e.g., $h / L_{0} \rightarrow 1$ ), the spatial resolution is comparative to the length scale of the flow structure, and the truncation error due to the discrete scheme is the dominant error source (blue patched regime). When the spatial resolution is even larger (e.g., $h / L_{0} \gg 1$ ), the insufficient sampling lower than the Nyquist frequency causes aliasing and unreliable, or more precisely, meaningless pressure field reconstructions.

The normalized histograms of the error level in the calculated pressure field at three different values of the spatial resolution as indicated in Fig. 2(a) (marked by orange, green, and blue frames), are shown 


\begin{tabular}{ccc}
\hline Parameters & Value & Unites \\
\cline { 2 - 3 }$H$ & $10^{2}$ & {$\left[\mathrm{~m}^{2}\right]$} \\
$\rho$ & $10^{3}$ & {$\left[\mathrm{~kg} / \mathrm{m}^{3}\right]$} \\
$v$ & $10^{-6}$ & {$\left[\mathrm{~m}^{2} / \mathrm{s}\right]$} \\
$t$ & 1,250 & {$[\mathrm{sec}]$} \\
\hline Characteristic scales & & \\
$L_{0}=\sqrt{2 v t}$ & 0.05 & {$[\mathrm{~m}]$} \\
$U_{0}=\frac{H}{2 \pi 0_{0} t}$ & 0.255 & {$[\mathrm{~m} / \mathrm{s}]$} \\
$p_{0}=\rho U_{0}^{2}$ & 64.85 & {$[\mathrm{~Pa}]$} \\
\hline Dimensionalized variables & & \\
{$[x \times y]$} & {$[-0.15,0.15] \times[-0.15,0.15]$} & {$[\mathrm{m}]$} \\
$u_{\text {peak }}$ & 0.0772 & {$[\mathrm{~m} / \mathrm{s}]$} \\
$p_{\text {peak }}-p_{\infty}$ & -8.106 & {$[\mathrm{~Pa}]$} \\
\hline Non-dimensionalized variables & & \\
{$[x \times y] / L_{0}$} & {$[-3,3] \times[-3,3]$} & - \\
$u_{\text {peak }} / U_{0}$ & 0.3033 & - \\
$\left(p_{\text {peak }}-p_{\infty}\right) / P_{0}$ & -0.125 & - \\
\hline
\end{tabular}

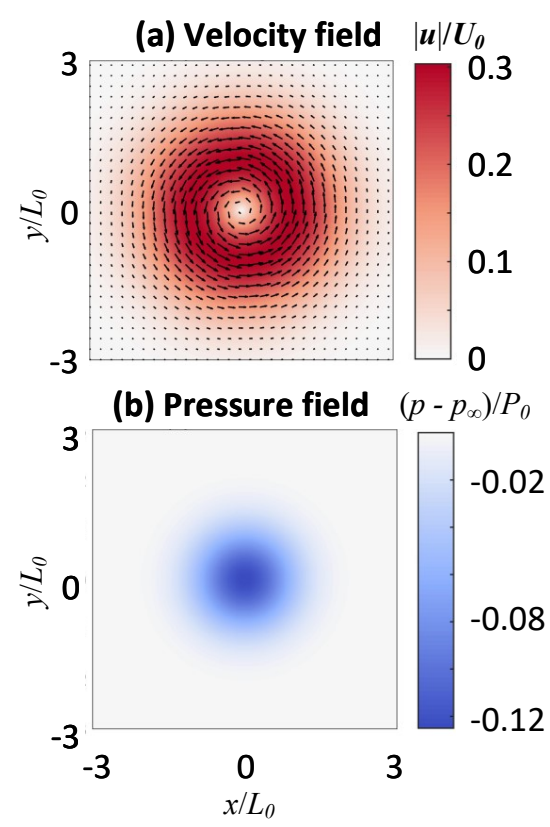

Figure 1: Parameter space for the numerical experiments (table to the left) and 2D visualization of the non-dimensional flow field in a box. (a) Velocity quiver plot over the magnitude and (b) the pressure field.

in Fig. 2(b-d), respectively. These histograms are normalized by $\left\|\varepsilon_{p}\right\|_{L^{2}(\Omega)} / P_{0} \times 100 \%$. They represent the probability density function (PDF) of the relative error (percentage compared to the characteristic pressure). One of the error fields in the reconstructed pressure drawn from the 5,000 independent numerical experiments for the three typical spatial resolutions are shown in Fig. 2(e-g), respectively.

More general validations can be achieved by varying the error level in the velocity field (e.g., different $\sigma_{u}^{2}$ and $\sigma_{v}^{2}$ ) and adjusting the flow field (e.g., a flow with different characteristic scales). We consider i) the same flow used in the above example (Fig. 1), but with larger error with different statistics (i.e., $\varepsilon_{u} \sim$ $\mathcal{N}\left(0, \sigma_{u}^{2}\right), \varepsilon_{v} \sim \mathcal{N}\left(0, \sigma_{v}^{2}\right)$, where $\sigma_{u} / U_{0}=1.57 \times 10^{-2}$ and $\sigma_{v} / U_{0}=3.93 \times 10^{-3}$, called setup 2 hereafter $)$;i) the younger stage $(t=312.5 \mathrm{sec})$ of the same decaying vortex (see the able in Fig. 1 for detailed parameters) in the same dimensional domain (meaning a larger non-dimensional size of the domain), and the same dimensional error level as setup 1 (i.e., $\varepsilon_{u} \sim N\left(0, \sigma_{u}^{2}\right), \varepsilon_{v} \sim N\left(0, \sigma_{v}^{2}\right)$, where $\sigma_{u} / U_{0}=\sigma_{v} / U_{0}=0.98 \times 10^{-3}$, called setup 3 hereafter).

Table 2: Parameters of two different flows for validation (setup $1 \& 2$, and a younger vortex for setup 3).

\begin{tabular}{cccc}
\hline Parameters & Setup 1 \& 2 & Setup 3 & Units \\
\hline$L_{0}=\sqrt{2 v t}$ & 0.05 & 0.025 & {$[\mathrm{~m}]$} \\
$U_{0}=\frac{H}{2 \pi L_{0} t}$ & 0.25 & 2.04 & {$[\mathrm{~m} / \mathrm{s}]$} \\
$P_{0}=\rho U_{0}^{2}$ & 64.85 & 4,150 & {$[\mathrm{~Pa}]$} \\
$u_{\text {peak }}$ & 0.1 & 0.87 & {$[\mathrm{~m} / \mathrm{s}]$} \\
$p_{\text {peak }}-p_{\infty}$ & -8.1 & -518.8 & {$[\mathrm{~Pa}]$} \\
$\operatorname{Re}$ & $1.27 \times 10^{4}$ & $5.0 \times 10^{4}$ & - \\
\hline
\end{tabular}

Similar numerical experiments are conducted and the results are shown in Fig. 3(a). The results from the numerical experiments agree with the theoretical predictions well for various flows and PIV error statistics. Comparing setup 1 and 2, which share the same flow field but different error statistics in the velocity field, we note that when the spatial resolution is large (e.g., larger than the optimal resolution of the setup 2, marked by the green circle in Fig. 3(a)), the truncation error dominates and the numerical experimental results collapse onto the same dashed line, which is solely determined by the nature of the flow. When the spatial resolution is small, the error in the velocity field from the PIV measurements is the major contributor to the error in the calculated pressure field. Thus, setup 2 introduces more error than setup 1, and the 


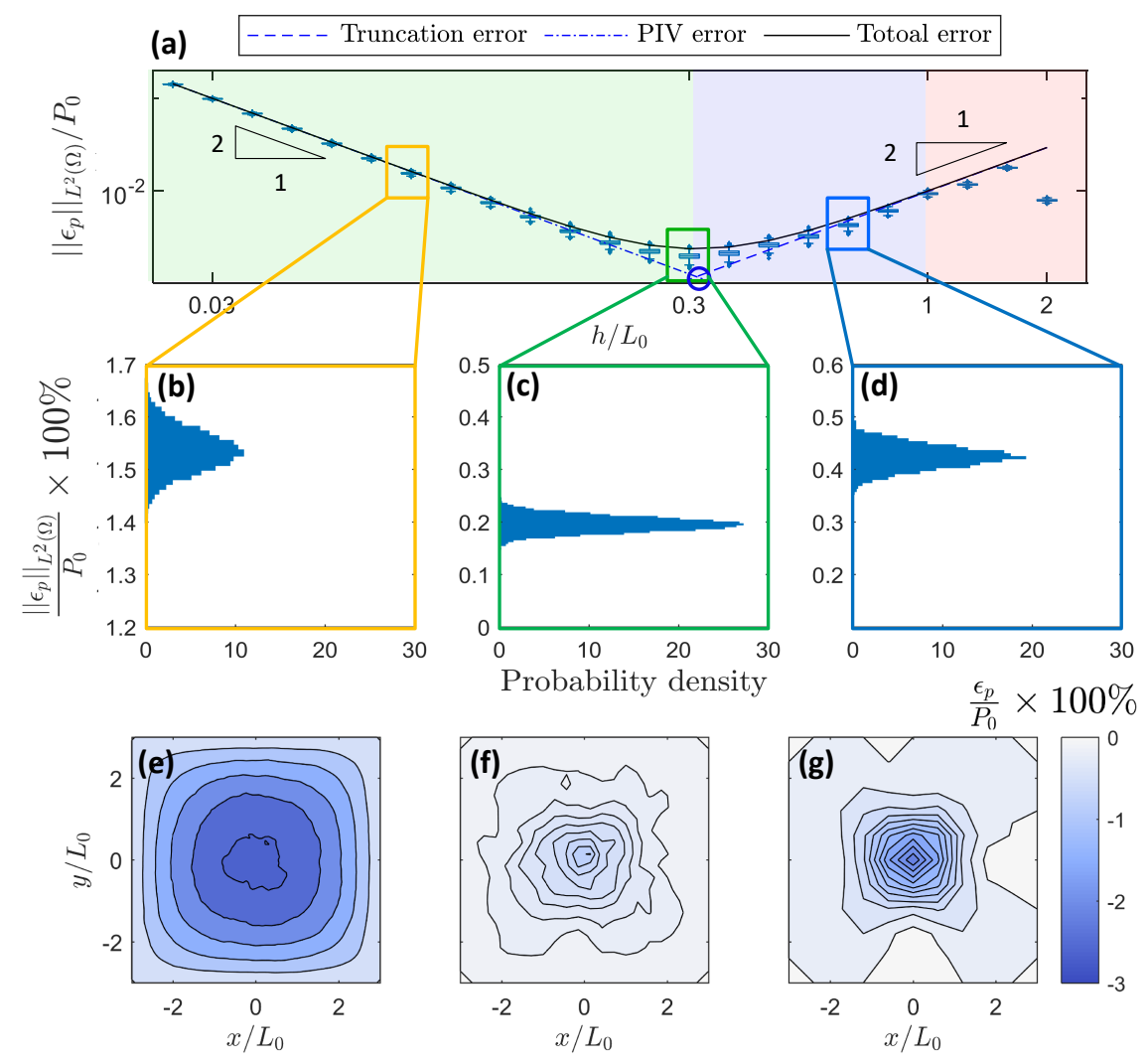

Figure 2: Error level in the calculated pressure field vs. spatial resolution. (a) Box plot of the error level in the calculated pressure field. Each box represents 5000 independent simulations. The green region is dominated by the error from the PIV measurements due to the spatial resolution being too fine. The blue region is the regime where the truncation error dominates because the resolution is too coarse. The red region indicates aliasing due to insufficient sampling lower than the Nyquist frequency. The dashed line represents the theoretical prediction of the truncation error, and the dash-dot line indicates the theoretical contribution from PIV measurement errors in the velocity field. The solid line represents the theoretical prediction of the total error in the calculated pressure field. (b-d) Normalized histograms of the relative error in the calculated pressure field for typical spatial resolutions (corresponding to the orange, green, and blue frames in Fig. 2(a), respectively). (e-g) Relative error field in pressure at several spatial resolutions (corresponding to $(\mathrm{b}-\mathrm{d})$, respectively.)

optimal spatial resolution is coarser than it is for setup 1 (the green circle is on the right of the blue circle). Comparing setup 1 and 3, a smaller characteristic length (radius of the vortex) of the flow in setup 3 implies that the optimal resolution for setup 3 is finer than setup 1 or 2 since a smaller scale flow structure must be resolved (the red circle is on the left of the green and blue circles). We emphasize that the vertical axis in Fig. 3(a) is a non-dimensional error level, not the dimensional value. Instead, $\left\|\varepsilon_{p}\right\|_{L^{2}(\Omega)} / P_{0}$ is a "error level" comparing the error to the corresponding characteristic pressure. Noting that setup 3 has significantly higher characteristic pressure than setup 1 and 2, it is not surprising that the minimum error level (or sensitivity of the pressure measurement) for setup 3 is lower than the other two setups (the red circle is located lower than the green and blue ones). However, this does not necessarily mean that the absolute pressure sensitivity for setup 3 is low. A more intuitive presentation can be found in Fig. 3(b), which is reconstructed from Fig. 3(a), but with physical dimensions included.

Figure 3(b) shows the error in the calculated pressure field versus spatial resolution for setup 1 (blue), 2 (green), and 3(red). When the spatial resolution is small (e.g., to the left of the red circle in Fig. 3(b)), the numerical experimental results (blue boxes and the red boxes) are collapsed onto the same dash-dot line because the same error statistics are shared as well as the same domain properties (e.g., size of the domain, type of BCs, etc.). The error in setup 2 is higher than that from setups 1 and 3 when the spatial resolution is small due to the larger random error in the velocity field. When the spatial resolution is high (e.g., to the 

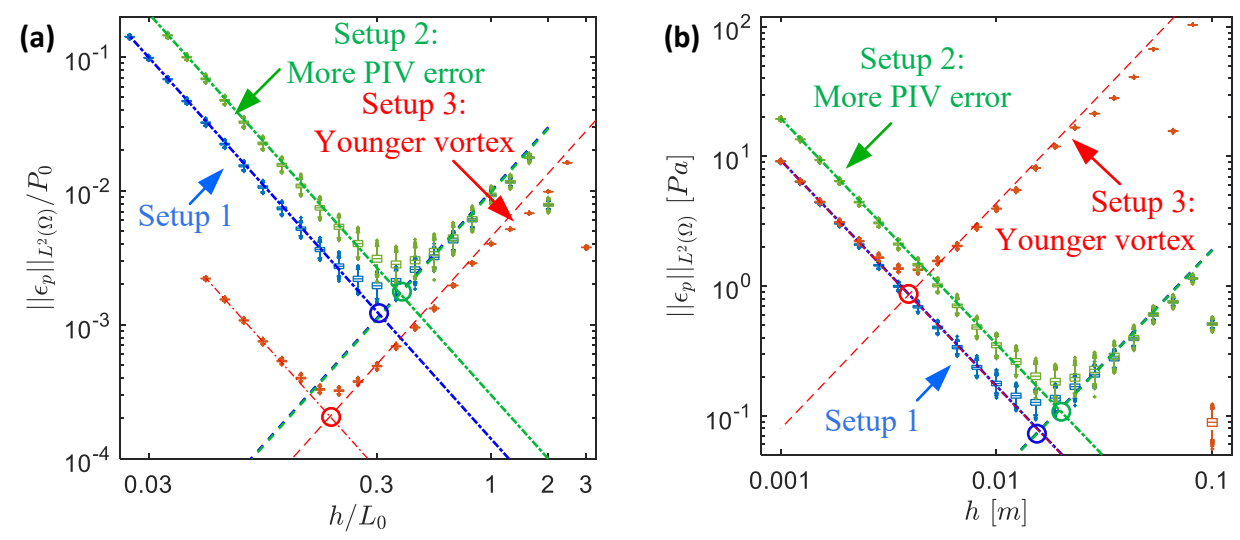

Figure 3: Non-dimensional (a) and dimensional (b) error in the calculated pressure field vs. non-dimensional spatial resolution. Numerical experiments of setup 1 (blue), 2 (green), and 3 (red). The dashed lines indicate the contribution from truncation error, and the dash-dot lines indicate the contribution of the error from the PIV measurement in the velocity field. The optimal spatial resolutions are marked by the circles at the intersections of the dashed lines and the dash-dot lines, with corresponding color schemes.

right of the green circle), the numerical experimental results from setup 1 and 2 (blue and green boxes) are collapsed onto the same theoretical prediction since they have the same flow field, despite these two setups have different error statistics in the velocity field.

More importantly, Fig. 3(b) clarifies how the flow field and error in the PIV measurements affect the optimal spatial resolution and the pressure reconstruction sensitivity (note the vertical positions of the colored circles) for the three different setups. The larger error in the PIV measurements requires coarser optimal spatial resolution and leads to lower pressure reconstruction sensitivity (comparing the positions of blue and the green circle). The smaller dominant flow structures in the flow require finer spatial resolution, however, leading to worse minimum resolvable pressure (comparing the positions of blue and the red circle).

A qualitative illustration of how the error from the PIV experimental measurement and the truncation error from the numerical solver compete against each other for the optimal spatial resolution, and at the same time, contribute together to the minimum error in the pressure field is shown in Fig. 4. Larger truncation error (e.g. due to a flow with higher spatial frequency) would shift the dashed lines up (Fig. 4(a)) and lead to a requirement for finer spatial resolution to achieve the minimum error in the pressure field (see the locus marked by the red circles and arrow head in Fig. 4(a)). More error in the velocity field from the PIV experiments will shift the dash-dot line up and require coarser spatial resolution for the minimum error in the calculated pressure field (see the locus marked by the red circles and arrow head in Fig. 4(b)). Based on the above observations, an intuitive impression is that one of the most challenging PIV experimental results for PIV-based pressure reconstruction is a flow with small scale dominant structures (usually leading to small characteristic length scales and more significant contributions from the truncation error) and high uncertainties in velocity field.

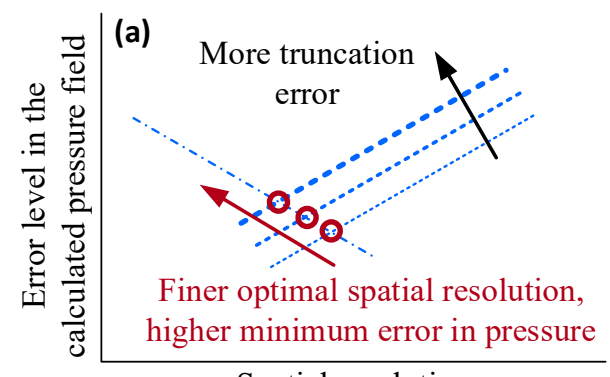

Spatial resolution

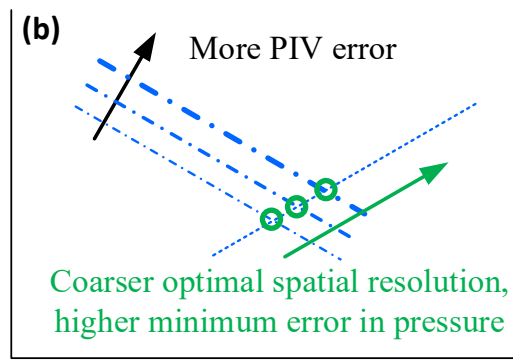

Spatial resolution

Figure 4: Qualitative illustration of the contributions and/or competition of the truncation error and PIV error. More truncation error in the domain leads to finer optimal spatial resolution, and higher minimum error in the calculated pressure field (marked by the red circles and arrow head in (a)). More error from the PIV experiments leads to coarser optimal resolution and higher minimum error in the pressure field. 


\section{Discussion and conclusions}

We have provided a rigorous framework that decouples the contribution of numerical truncation and experimental error to the pressure field reconstructed from PIV experiments. Based on this framework, we point out that the error propagation from the PIV-based velocity field measurements to the calculated pressure field is affected by many factors. For instance, beyond the quality of the PIV experiments, the geometry and boundary conditions of the domain, the physical profile of the flow, and the numerical scheme (e.g., grid spacing) of the pressure solver play a significant role. In this paper we have focused on one of these factors: how the spatial resolution of the velocity vector field from PIV impacts the error propagation. Specifically, we give a precise description of the competition between the truncation error from the numerical schemes and the experimental error from PIV experiments over the different spatial resolutions. When the spatial resolution is relatively fine, the error from the experimental data dominates the error propagation and when the spatial resolution is relatively coarse, truncation error due to the numerical scheme of the pressure solver governs the error propagation. Thus there is an optimal spatial resolution that minimizes the error propagation of a given flow. The corresponding minimum field-wide error level in the calculated pressure field can be considered the minimum resolvable pressure for the calculated field, or the effective sensitivity of the reconstructed pressure field.

We emphasize that the current research mainly focuses on a general framework that decouples error from the true value in the calculated pressure field. The uncertainties in the calculated pressure field can then be directly analyzed. Although the framework in this work is general, the specific form of some of the pertinent equations (e.g., (3)) depends on the specific numerical schemes of the solver (e.g., second order central finite difference with structural grid spacing for the current consideration), and the error model (e.g., point-wise Gaussian noise at each grid point) is not general. Different numerical schemes and more sophisticated models of the velocity field PIV-measure error will not fundamentally change the main results discussed above, and the approach taken here provides a guide for future investigations of such setups.

\section{References}

Azijli I, Sciacchitano A, Ragni D, Palha A, and Dwight RP (2016) A posteriori uncertainty quantification of PIV-based pressure data. Experiments in Fluids 57:1-15

Charonko JJ, King CV, Smith BL, and Vlachos PP (2010) Assessment of pressure field calculations from particle image velocimetry measurements. Measurement Science and Technology 21:105401

Charonko JJ and Vlachos PP (2013) Estimation of uncertainty bounds for individual particle image velocimetry measurements from cross-correlation peak ratio. Measurement Science and Technology 24:065301

Coleman HW and Steele WG (2009) Experimentation, validation, and uncertainty analysis for engineers. John Wiley \& Sons, Hoboken, NJ

de Kat R and Van Oudheusden B (2012) Instantaneous planar pressure determination from PIV in turbulent flow. Experiments in fluids 52:1089-1106

Ghaemi S, Ragni D, and Scarano F (2012) PIV-based pressure fluctuations in the turbulent boundary layer. Experiments in fluids 53:1823-1840

He C, Liu Y, and Gan L (2020) Instantaneous pressure determination from unsteady velocity fields using adjoint-based sequential data assimilation. Physics of Fluids 32:035101

Jeon Y, Earl T, Braud P, Chatellier L, and David L (2016) 3d pressure field around an inclined airfoil by tomographic TR-PIV and its comparison with direct pressure measurements. in 18th international symposium on the application of laser techniques to fluid mechanics. Lisbon, Portugal. pages 4-7

Jeon YJ, Chatellier L, Beaudoin A, and David L (2015) Least-square reconstruction of instantaneous pressure field around a body based on a directly acquired material acceleration in timeresolved PIV, 11th int. in Symp. Part. Image Velocim.-PIV15

Léon O, Piot E, Sebbane D, and Simon F (2017) Measurement of acoustic velocity components in a turbulent flow using LDV and high-repetition rate PIV. Experiments in Fluids 58:72 
Lignarolo L, Ragni D, Krishnaswami C, Chen Q, Ferreira CS, and Van Bussel G (2014) Experimental analysis of the wake of a horizontal-axis wind-turbine model. Renewable Energy 70:31-46

Liu X and Katz J (2006) Instantaneous pressure and material acceleration measurements using a fourexposure PIV system. Experiments in Fluids 41:227-240

Matthew Faiella JPW Corwin G J Macmillan and Pan Z (2021) Error propagation dynamics of velocimetrybased pressure field calculations (2): on the error profile. Measurement Science and Technology 26:074002

McClure J and Yarusevych S (2017) Optimization of planar PIV-based pressure estimates in laminar and turbulent wakes. Experiments in Fluids 58:62

Pan Z (2016) Error Propagation Dynamics of PIV-based Pressure Field Calculation. Ph.D. thesis. Brigham Young University

Pan Z, Whitehead J, Thomson S, and Truscott T (2016) Error propagation dynamics of PIV-based pressure field calculations: how well does the pressure Poisson solver perform inherently?. Measurement Science and Technology 27:084012

Pan Z, Whitehead JP, Richards G, Truscott TT, and Smith BL (2018) Error propagation dynamics of pivbased pressure field calculation (3): What is the minimum resolvable pressure in a reconstructed field?. arXiv preprint arXiv: 180703958

Panton RL (2006) Incompressible flow. John Wiley \& Sons, Hoboken, NJ

Porfiri M and Shams A (2017) New york university brooklyn, brooklyn, ny, united states. Dynamic Response and Failure of Composite Materials and Structures page 395

Pröbsting S, Scarano F, Bernardini M, and Pirozzoli S (2013) On the estimation of wall pressure coherence using time-resolved tomographic PIV. Experiments in fluids 54:1-15

Scarano F (2012) Tomographic PIV: principles and practice. Measurement Science and Technology 24:012001

Schwabe M (1935) Über druckermittlung in der nichtstationären ebenen strömung. Ingenieur-Archiv 6:3450

Sciacchitano A, Wieneke B, and Scarano F (2013) PIV uncertainty quantification by image matching. Measurement Science and Technology 24:045302

Van Gent PL, Van Oudheusden BW, and Schrijer FF (2018) Determination of mean pressure from piv in compressible flows using the reynolds-averaging approach. Experiments in Fluids 59:1-14

Van Oudheusden B (2013) PIV-based pressure measurement. Measurement Science and Technology 24:032001

Van Oudheusden BW, Scarano F, Roosenboom EW, Casimiri EW, and Souverein LJ (2007) Evaluation of integral forces and pressure fields from planar velocimetry data for incompressible and compressible flows. Experiments in Fluids 43:153-162

Wang CY, Gao Q, Wei RJ, Li T, and Wang JJ (2017) Spectral decomposition-based fast pressure integration algorithm. Experiments in Fluids 58:84

Wieneke B (2015) Piv uncertainty quantification from correlation statistics. Measurement Science and Technology 26:074002

Zhang C, Wang J, Blake W, and Katz J (2017) Deformation of a compliant wall in a turbulent channel flow. Journal of Fluid Mechanics 823:345-390 